\title{
Blind Wide Area Surveys: Where will we find redshifted atomic and molecular absorption?
}

\author{
Stephen J. Curran* \\ University of New South Wales \\ E-mail: Sjclphys.unsw.edu.au \\ Matthew T. Whiting \\ CSIRO Australia Telescope National Facility \\ E-mail: Matthew.Whitingecsiro.au \\ John K. Webb \\ University of New South Wales \\ E-mail: jkw@phys.unsw.edu.au
}

Spectroscopy of redshifted radio absorption of atomic and molecular species provide excellent probes of the cold component of the gas in the early Universe which can be used to address many important issues, such as measuring baryonic content, probing large-scale structure and galaxy evolution, as well as obtaining independent measurements of various combinations of fundamental constants at large look-back times. However, such systems are currently very rare with only 80 detected in $\mathrm{H}$ I $21-\mathrm{cm}$ and five in $\mathrm{OH}$ and millimetre-band species. Here we summarise the main selection criteria responsible for this and how the next generation of radio telescopes are expected to circumvent these through their wide instantaneous bandwidths and fields-of-view. Specifically:

1. H in absorbers occulting distant quasars: These are usually found in known optical absorbers and wideband radio surveys could reveal a much fainter population. However, the 21-cm absorption strength may be correlated with the width of the singly ionised metal species, suggesting that these may be weak, and due to the effects of a flat expanding Universe on the covering factor, we expect the highest detection rates at $z<1$.

2. H I absorption associated with the host galaxy/quasar: Due to high degrees of ionisation/excitation rendering $21-\mathrm{cm}$ undetectable near active nuclei with ultra-violet luminosities of $L_{\mathrm{UV}} \gtrsim 10^{23} \mathrm{~W} \mathrm{~Hz}^{-1}$, future searches should be magnitude limited, e.g. at $z>1$, blue magnitudes should be $B \gtrsim 19$ with $z>2 \Rightarrow B \gtrsim 21$ and $z>3 \Rightarrow B \gtrsim 22$.

3. $\mathrm{OH}$ (and millimetre-band) absorption: For all of the known redshifted molecular absorption systems a correlation is found between the molecular fraction and the optical-nearinfrared colour $(V-K)$, with the five known $\mathrm{OH}$ absorbers all having $V-K \gtrsim 5$. Therefore spectral scans towards extremely red radio sources are expected to uncover any dusty intervening, molecular rich absorbers reponsible for the obscuration of the optical light.

Panoramic Radio Astronomy: Wide-field 1-2 GHz research on galaxy evolution

June 2-5 2009

Groningen, the Netherlands

\footnotetext{
* Speaker.
} 


\section{Optically Selected Intervening Absorbers}

With total neutral hydrogen column densities of $N_{\mathrm{HI}} \gtrsim 10^{20} \mathrm{~cm}^{-2}$ and precisely determined redshifts, the detection of $\mathrm{H}$ I $21-\mathrm{cm}$ and $\mathrm{OH}$ in damped Lyman- $\alpha$ absorption systems (DLAs) should be like shooting fish in a barrel. However, there are only 40 cases, out of 151 published searches (see [1]), of 21-cm being detected in redshifted intervening absorbers (Lyman- $\alpha$ and $\mathrm{Mg}$ II) and there has never been a detection of molecular absorption in either the decimetre $(\mathrm{OH})$ or millimetre band $\left(\mathrm{CO}, \mathrm{HCO}^{+}\right.$, etc., summarised in [2] $)$.

\subsection{Absorption by Atomic Gas}

In an absorption system the neutral hydrogen column density is related to the $21-\mathrm{cm}$ line strength via $N_{\mathrm{HI}}=1.823 \times 10^{18} T_{\text {spin }} \int \tau d v$, where $T_{\text {spin }}$ is the mean harmonic spin temperature of the gas and $\int \tau d v$ is the velocity integrated optical depth of the line. The optical depth is defined via $\tau \equiv-\ln \left(1-\frac{\sigma}{f S}\right)$, where $\sigma$ is the depth of the line and $S$ and $f$ the flux density and covering factor of the background continuum source, respectively. Therefore in the optically thin regime, the expression simplifies to $N_{\mathrm{HI}}=1.823 \times 10^{18} \frac{T_{\mathrm{spin}}}{f} \int \frac{\sigma}{S} d v$ and if the Lyman- $\alpha$ (from which $N_{\mathrm{HI}}$ is determined) and $21-\mathrm{cm}$ absorption arise in the same sight-line, the velocity integrated "optical depth", $\int \frac{\sigma}{S} d v$, gives the ratio of the spin temperature to the covering factor, $T_{\text {spin }} / f$.

Since the $21-\mathrm{cm}$ detections occur overwhelmingly at $z_{\text {abs }} \lesssim 1$, where there is a mix of detections and non-detections, [10] advocate a scenario where low redshift absorbers have a mix of spin temperatures, while those at high redshift exhibit exclusively high temperatures. This result, however, relies on the $z_{\mathrm{abs}}>2$ absorbers having the maximum permissible covering factor $(f=1)$, which yields the maximum possible spin temperature [3]. This results in a huge disparity in the values, specifically $T_{\text {spin }} \approx 200$ to $\approx 9000 \mathrm{~K}$ [10]. Furthermore, with a maximum possible spin temperature of $T_{\text {spin }} /(f=1) \approx 140 \mathrm{~K}$ at $z_{\text {abs }}=2.289$ [11], the DLA towards TXS 0311+430 rules out the hypothesis that a high redshift necessarily entails a high spin temperature.

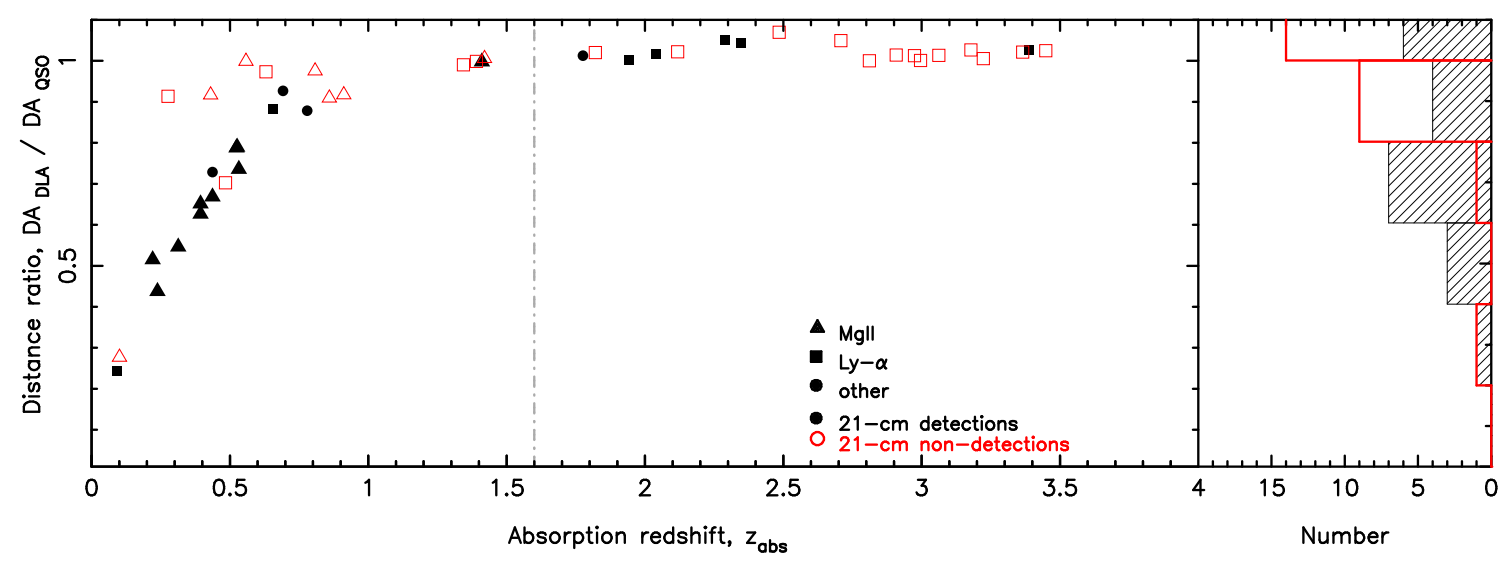

Figure 1: The angular diameter distance ratio versus redshift for the DLAs searched in 21-cm absorption. The filled symbols/hatched histogram represent the $21-\mathrm{cm}$ detections and the unfilled symbols/unfilled histogram the non-detections. The symbol shapes designate the transition through which the absorber was discovered. Updated from [5].

Although without high resolution imaging of the absorber and the background emission region at the redshifted $21-\mathrm{cm}$ frequency the $T_{\text {spin }} / f$ degeneracy cannot be broken, we can suggest that the 
covering factor may nonetheless play an important rôle. This is apparent in Fig. 1, where we show the ratio of the angular diameter distances to the absorber and quasar against redshift: Due to the effects of a flat expanding Universe, beyond $z \approx 1.6$ the angular diameter distance decreases with redshift and so, whereas low redshift systems may exhibit a variety of $D A_{\mathrm{DLA}} / D A_{\mathrm{QSO}}$ ratios, high redshift absorbers will always have $D A_{\mathrm{DLA}} / D A_{\mathrm{QSO}} \approx 1$.

That is, at $z_{\mathrm{abs}} \gtrsim 1$ the absorber and quasar are essentially at the same angular diameter distance and so for given absorber and emitter cross-sections, these absorbers will cover the background emission much less effectively that those with low $D A_{\mathrm{DLA}} / D A_{\mathrm{QSO}}$ ratios. The mix at low redshift arises from the mix of low and high redshift background quasars, with the overall distribution following the same pattern as that of the "spin temperature" distribution of [10] - a variety at low redshift and exclusively high values at high redshift. Although in this case it is a covering factor effect, which is not subject to the same assumptions.

This can also explain the high detection rate in DLAs originally found through the Mg II, rather than the Lyman- $\alpha$, line: The Mg II doublet can be observed by ground-based telescopes at redshifts of $0.2 \lesssim z_{\text {abs }} \lesssim 2.2$, although the Lyman- $\alpha$ transition is restricted to $z_{\text {abs }} \gtrsim 1.7$. That is, $\mathrm{Mg}$ II observations preferentially select low redshift absorbers, thus not resulting in exclusively high $D A_{\mathrm{DLA}} / D A_{\mathrm{QSO}}$ ratios (and hence lower covering factors). On the subject of $\mathrm{Mg}$ II absorption,

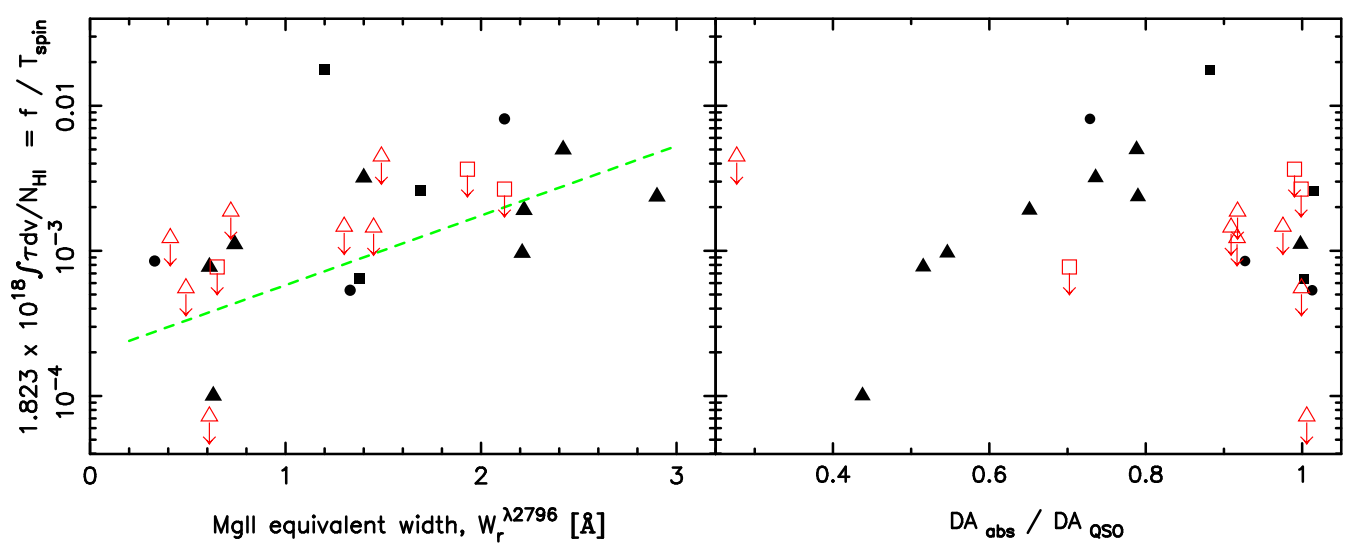

Figure 2: The 21-cm line strength versus the rest frame equivalent width of the Mg II $2796 \AA$ line (left) and the angular diameter distance ratio (right) for the DLAs searched in 21-absorption. The correlation shown by the least-squares fit in the left panel is significant at $2.52 \sigma$ (see [1]).

邨 find a correlation between the 21-cm line strength and the Mg II $2796 \AA$ A equivalent width, although there are also DLAs which span similar equivalent widths while remaining undetected in 21-cm (Fig. 2. left). However, of the nine which have good limits, eight of the non-detections have $D A_{\mathrm{DLA}} / D A_{\mathrm{QSO}} \gtrsim 0.9$ (Fig. 2, right), again suggesting a strong covering factor effect due to the line-of-sight geometry.

\subsection{Absorption by Molecular Gas}

Although the $\mathrm{H}_{2}$ molecule has been detected through the Lyman and Werner ultra-violet bands ${ }^{1}$ in 17 DLAs, extensive millimetre-wave band searches have yet to yield a detection, despite exceeding the sensitivities to required to detect the known millimetre/OH absorbers by an

\footnotetext{
${ }^{1}$ Redshifted into the optical-band at $z_{\mathrm{abs}} \gtrsim 1.7$.
} 
order of magnitude ([2] and references therein). We have noted [6], however, that these radio-band absorbers have molecular fractions $\mathscr{F} \equiv \frac{2 N_{\mathrm{H}_{2}}}{2 N_{\mathrm{H}_{2}}+N_{\mathrm{HI}}} \approx 0.7-1$ and optical-near-infrared colours of $V-K \gtrsim 5$, whereas the optical-band absorbers have $\mathscr{F} \sim 10^{-7}-0.3$ and $V-K \lesssim 4$ (Fig. 3). This
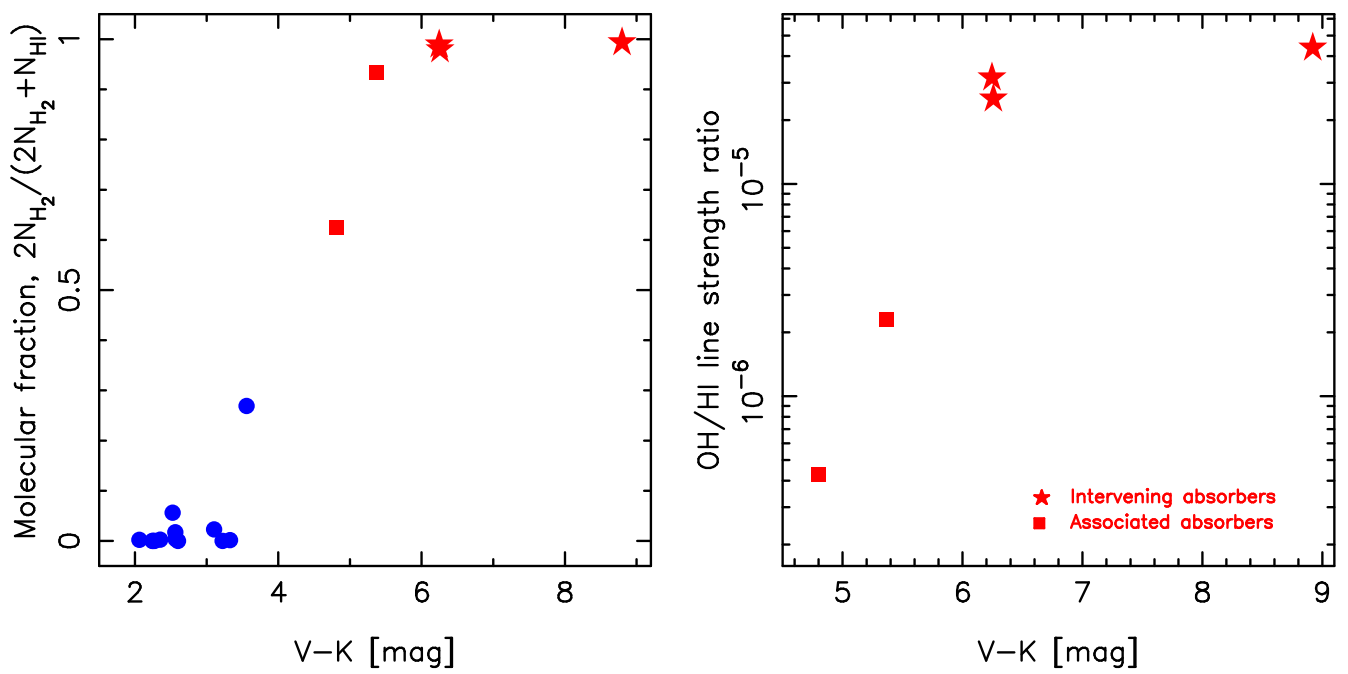

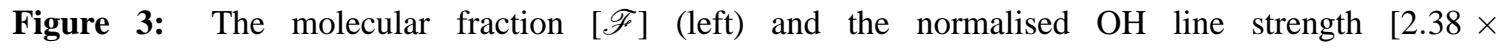
$\left.10^{14} \int \tau_{\mathrm{OH}} d v / 1.82 \times 10^{18} \int \tau_{\mathrm{HI}} d v\right]$ (right) versus the optical-near infrared colour. The circles represent the $\mathrm{H}_{2}$-bearing DLAs (all intervening absorbers) and the squares and stars the $\mathrm{OH}$ absorbers. Updated from [6], where the correlation between $\mathscr{F}$ and $V-K$ is now significant at $3.7 \sigma$.

is strong evidence that quasar light is reddened by the dust in the foreground absorber, which is necessary to prevent the dissociation of the molecular gas by the ambient ultra-violet field.

\section{Radio Selected Absorbers}

\subsection{Intervening Absorption}

From the above discussion (Sect. 1.2), it is apparent that DLAs are simply too blue to indicate a sufficiently large column of dust conducive to molecular abundances which can be detected by current radio telescopes, although the SKA will be capable of detecting normalised $\mathrm{OH}$ column densities of $\frac{N_{\mathrm{OH}}}{N_{\mathrm{HI}}} \lesssim 10^{-6.5} \frac{f_{\mathrm{OH}}}{f_{\mathrm{HI}}} \frac{T_{\mathrm{spin}}}{T_{\mathrm{ex}}}$ (at $V-K \lesssim 5$, Fig. B). In the meantime, searches for $18-\mathrm{cm} \mathrm{OH}$ absorption should be targetted towards very red, radio-loud objects. However, this very redness means that there are generally no optical redshifts of any absorption features towards these quasars, which in turn means that we have no knowledge of which frequency to tune the receivers.

Therefore spectral scans of the entire redshift space towards red quasars are required in order to detect the absorber responsible for the obscuration of the optical light. Although they will not be any more sensitive than current state-of-the-art radio telescopes, the large instantaneous bandwidths (e.g. $300 \mathrm{MHz}$ with the ASKAP) will make the forthcoming SKA pathfinders ideal instruments with which to undertake such spectral scans. Furthermore, the large fields-of-view (e.g. 30 square degrees with the ASKAP) will facilitate truly blind surveys, where full range spectra $\left(0 \leq z_{\mathrm{HI}} \lesssim 1.0\right.$ and $0 \leq z_{\mathrm{OH}} \lesssim 1.4$ ) of large areas of sky may be obtained in a few re-tunings. 


\subsection{Associated Absorption}

Although we may have to wait for the next generation of radio telescopes to undertake effective full spectral scans, we can currently use the redshift of the background radio source to search for absorption associated with the host galaxy/quasar. However, from a recent survey of $z_{\mathrm{em}} \approx 3-4$

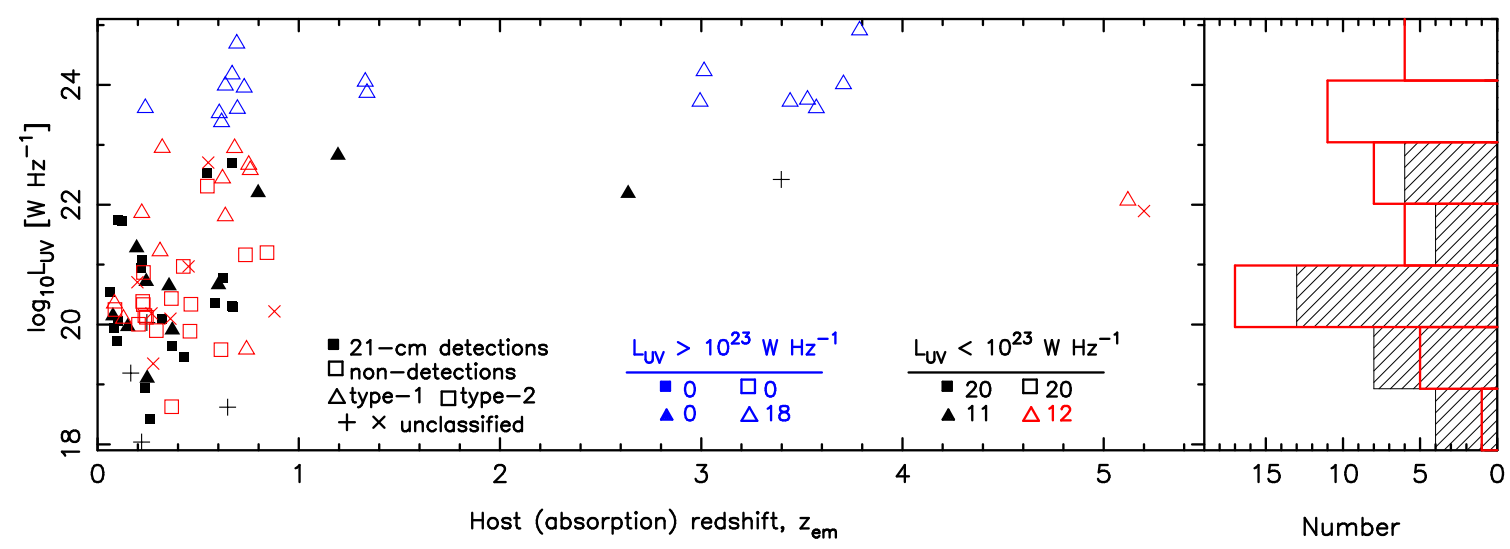

Figure 4: The $\lambda=1216 \AA$ luminosity versus the redshift for the radio galaxies and quasars searched in associated $21-\mathrm{cm}$ absorption. The symbol and histogram filling is as per Fig. 11 with the shapes representing the AGN classifications: Triangles - type- 1 , squares - type- 2 and $+\& x$ designating undetermined types for the detections and non-detections, respectively. Updated from [8].

radio sources for $\mathrm{H} \mathrm{I}$ and $\mathrm{OH}$, we did not detect any absorption in the hosts of any of these objects. Upon a thorough analysis of the optical photometry, we found that all of our targets have ultraviolet luminosities of $L_{\mathrm{UV}} \gtrsim 10^{23} \mathrm{~W} \mathrm{~Hz}^{-1}$ [8]. This could be interpreted as the by-product of some other selection effect which causes the paucity of $\mathrm{H} \mathrm{I} 21-\mathrm{cm}_{\text {absorption }}{ }^{2}$ at high redshift, if it were not for the fact we found this to apply at all redshifts (Fig. (4).

It is therefore apparent that we have identified a critical ultra-violet luminosity, above which the gas is excited to beyond the detection limits of current instruments. Higher UV luminosities can also account for the lower $21-\mathrm{cm}$ detection rates in quasars in comparison to radio galaxies [7], which is currently attributed to unified schemes of AGN, where quasars are predominantly type-1 objects and radio galaxies type- 2 (e.g. [9] and references therein). Although, all of the $L_{\mathrm{UV}} \gtrsim 10^{23}$ $\mathrm{W} \mathrm{Hz}{ }^{-1}$ are indeed type-1, it is the inclusion of these which gives the apparent bias against type-1 objects, with a $50 \% 21-\mathrm{cm}$ detection rate being found for both AGN types at $L_{\mathrm{UV}} \lesssim 10^{23} \mathrm{~W} \mathrm{~Hz}^{-1}$ (Fig. (1). Note also that, with the exclusion of the $L_{\mathrm{UV}} \gtrsim 10^{23} \mathrm{~W} \mathrm{~Hz}^{-1}$ sources, the detection rate in "compact objects" (gigahertz peaked spectrum and compact steep spectrum sources) is not any higher than for the remainder of the radio/galaxy sample [7].

Although the detection rate is only $50 \%$ at $L_{\mathrm{UV}} \lesssim 10^{23} \mathrm{~W} \mathrm{~Hz}^{-1}$ (probably due to the orientation of the galactic disk, [团]), we believe that searches for associated absorption will have to be restricted to these luminosities if detection rates of $>0 \%$ are to be obtained. As a search diagnostic, in Fig. 5 we show the blue magnitude against the redshift for all of the published $z_{\mathrm{em}} \gtrsim 0.1$ searches. From this we see that, although our previous $z_{\mathrm{em}} \approx 3-4$ survey was generally restricted to $B \gtrsim 19$, these

\footnotetext{
${ }^{2}$ In light of the $\mathrm{H}$ I non-detections it is not surprising that we did not detect $\mathrm{OH}$. In any case, all of the targets have $V-K \leq 2.6$, suggesting a low level of dust obscuration and thus low molecular fractions (Sect. 1.2).
} 


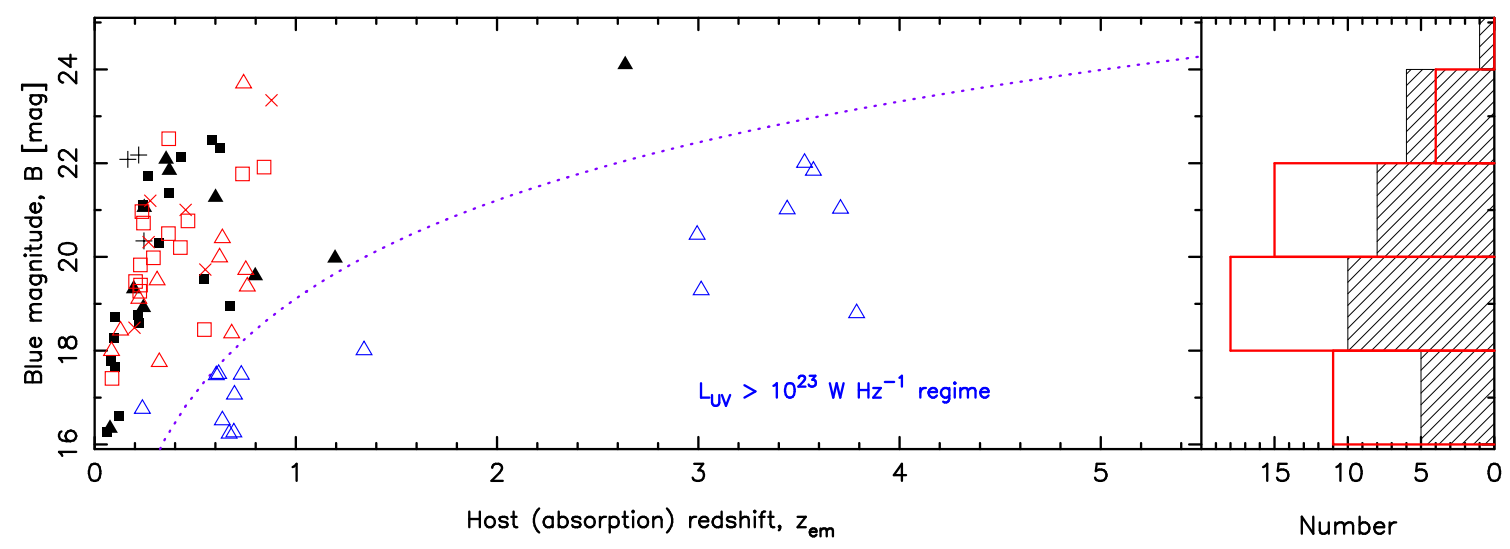

Figure 5: As Fig. 田, but showing the blue magnitude of the source on the ordinate. The curve shows which $B$ magnitude corresponds to $L_{\mathrm{UV}}=10^{23} \mathrm{~W} \mathrm{~Hz}^{-1}$ for a spectral slope of $\alpha=-1.5$.

magnitudes are still not sufficiently faint to indicate $L_{\mathrm{UV}} \lesssim 10^{23} \mathrm{~W} \mathrm{~Hz}^{-1}$ at such high luminosity distances. That is, once again, optical selection of targets introduces a bias against the detection of radio absorption lines, and blind wide-band searches over large fields-of-view are expected to significantly increase the number of $\mathrm{H} \mathrm{I}$ and $\mathrm{OH}$ absorbers at high redshift.

\section{References}

[1] Curran, S. J, On the detectability of H I 21 -cm in MgII absorption systems, MNRAS, accepted [arXiv:0910.3998]

[2] Curran, S. J., Murphy, M. T., Pihlström, Y. M., Webb, J. K., Bolatto, A. D. \& Bower, G. C., Molecular fraction limits in damped Lyman- $\alpha$ absorption systems, MNRAS 352563

[3] Curran, S. J., Murphy, M. T., Pihlström, Y. M., Webb, J. K. \& Purcell, C. R., Spin temperatures and covering factors for H I 21-cm absorption in damped Lyman- $\alpha$ systems, MNRAS 3561509

[4] Curran, S. J., Tzanavaris, P., Pihlström, Y. M. \& Webb, J. K., Relationships between the H I 21 -cm line strength, $M g$ II equivalent width and metallicity in damped Lyman- $\alpha$ absorption systems, MNRAS 3821331

[5] Curran, S. J. \& Webb, J. K., The detectability of H I 21 -cm absorption in damped Lyman- $\alpha$ systems, MNRAS 371356

[6] Curran, S. J., Whiting, M., Murphy, M. T., Webb, J. K., Longmore, S. N., Pihlström, Y. M., Athreya, R. \& Blake, C, A survey for redshifted molecular and atomic absorption lines I, MNRAS 371431

[7] Curran, S. J. \& Whiting, M. T, H I 21-cm absorption and unified schemes of active galactic nuclei, ApJ, submitted [arXiv:0902.3493]

[8] Curran, S. J., Whiting, M. T., Wiklind, T., Webb, J. K., Murphy, M. T. \& Purcell, C. R., A survey for redshifted molecular and atomic absorption lines II, MNRAS 391765

[9] Gupta, N. \& Saikia, D. J., Unification scheme and the distribution of neutral gas in compact radio sources, MNRAS $\mathbf{3 7 0} 738$

[10] Kanekar, N. \& Chengalur, J. N., A deep search for $21 \mathrm{~cm}$ absorption in high redshift damped Lyman- $\alpha$ systems, A\&A 399857

[11] York, B. A., Kanekar, N., Ellison, S. L. \& Pettini, M., Discovery of $21 \mathrm{~cm}$ absorption in a $z_{\mathrm{abs}}=2.289$ DLA towards TXS 0311+430: The first low spin temperature absorber at $z>1$, MNRAS 382 L53 